CERN-TH/95-37

DFTT-14/95

\title{
VON NEUMANN AND SHANNON-WEHRL ENTROPY FOR SQUEEZED STATES AND COSMOLOGICAL PARTICLE PRODUCTION
}

\author{
M. Gasperini \\ Dipartimento di Fisica Teorica, Via P.Giuria 1, 10125 Turin, Italy \\ and \\ M. Giovannini \\ Theory Division, CERN, CH-1211, Geneva 23, Switzerland
}

\begin{abstract}
We show that the effective coarse graining of a two-mode squeezed density matrix, implicit in the Wehrl approaches to a semiclassical phase-space distribution, leads to results in agreement with previous different definitions of entropy for the process of pair production from the vacuum. We also present, in this context, a possible interpretation of the entropy growth as an amplification (due to the squeezing) of our lack of knowledge about the initial conditions, which gives rise to an effective decoherence of the squeezed density matrix.
\end{abstract}

To appear in the "String gravity and physics at the Planck energy scale" (World Scientific, Singapore, 1995)

CERN-TH/95-37

February 1995 


\title{
VON NEUMANN AND SHANNON-WEHRL ENTROPY FOR SQUEEZED STATES AND COSMOLOGICAL PARTICLE PRODUCTION
}

\author{
M. Gasperini and M. Giovannini \\ Dipartimento di Fisica Teorica, Universitá di Torino, \\ Via P.Giuria 1, 10125 Turin, Italy
}

\begin{abstract}
We show that the effective coarse graining of a two-mode squeezed density matrix, implicit in the Wehrl approaches to a semiclassical phase-space distribution, leads to results in agreement with previous different definitions of entropy for the process of pair production from the vacuum. We also present, in this context, a possible interpretation of the entropy growth as an amplification (due to the squeezing) of our lack of knowledge about the initial conditions, which gives rise to an effective decoherence of the squeezed density matrix.
\end{abstract}

Some years ago it was suggested that the in quantum process of pair production from the vacuum, induced by the action of a time-varying classical background, the growth of the average number of quanta of a given field should be naturally associated to the growth of entropy of that field $[1,2]$. More recently, such an entropy growth was quantified on the grounds of a squeezed state approach to the process of particle production [3-7]. With this approach one can easily compute, in particular, the total entropy $S$ stored in the cosmological perturbations amplified by inflation, and one obtains $[4,6]$

$$
S \simeq\left(\frac{H_{f}}{M_{p}}\right)^{3 / 2} S_{c m b}
$$

where $S_{c m b}$ is the thermal black-body entropy of the $3 K^{o}$ photon microwave background, $H_{f}$ the final curvature scale of inflation, and $M_{p}$ the Planck mass.

The use of the squeezed state formalism to compute entropy production might seem to give rise to a puzzle, since the squeezed evolution is unitary. The solution of the puzzle is that, of course, there is no loss of information in principle associated to the evolution of the initial state into a final squeezed quantum state 
(and so no possible information paradox, like in the case of black-hole dynamics). The loss of information occurs however in practice, as a consequence of the process of measure of the observables characterizing the final squeezed state. There are indeed variables (related to the so-called "superfluctuant" operators [8]) which are amplified by the squeezed evolution and become thus available for macroscopic observations, while other variables (the "subfluctuant" ones [8]) are correspondingly "squeezed" and become unobservable.

A simple and intuitive way to illustrate this situation is to consider a semiclassical description of the squeezing process as a parametric amplification of the wave function, "hitting" the effective potential barrier of a Schroedinger-like equation (with time-like variable) [9,10]. The final amplitude of the wave scattered by the barrier may be larger or smaller than the initial amplitude, depending on the phase with which the wave enters the barrier. What is macroscopically detected, however, is the average amplification of the wave, out of an initial random distribution of phases. This point was clearly stressed already in the first paper on the cosmological amplification of tensor perturbations [11]. A macroscopic observation of the quantum fluctuations of the metric, amplified by the cosmic evolution, traces out over phases.

In order to compute the entropy associated to a squeezing process, this macroscopic loss of information has to be taken into account in the form of a suitable "coarse graining" of the density matrix of the final (pure) squeezed state. Such a coarse graining was originally performed in a Fock basis, i.e. with respect to the eigenstates of the number operator, by averaging over phases [3-5]. The number operator, however, is only a particular example of superfluctuant variable which grows in the case of a pair production process, while the conjugate variable (the phase) is correspondingly squeezed. This suggests the use, in general, of the eigenstates of the superfluctuant operator as the natural basis for the coarse graining of a squeezed density matrix [6,7]. A possible different basis, that of coherent states with real amplitude, has been suggested however in [12].

It is important to stress that all these different approaches $[3-7,12]$ to the entropy of a squeezed state exactly lead to the same result when the entropy growth $\Delta S_{k}$, for each mode $k$, is evaluated in the large squeezing regime, namely

$$
\Delta S_{k} \simeq 2 r_{k}, \quad r_{k}>>1
$$

where $r_{k}$ is the modulus of the (complex) squeezing parameter [8]. In the previously quoted papers, however, the decoherence process which leads to a reduced 
density matrix $\rho_{\text {red }}$ (from which to compute the quantum Von Neumann entropy) is imposed "by hand", by setting the off-diagonal terms of the matrix to zero in some chosen basis; at best, like in the case of the number basis, it is justified in a semiclassical way using a stochastic approach and a sort of random-phase approximation $[3,4]$. The problem is that it is formally impossible in the number basis, as well as in the more general superfluctuant basis, to compute the quantum von Neumann entropy $\left(-T r \rho_{\text {red }} \ln \rho_{\text {red }}\right)$ from a reduced matrix $\left(\rho_{\text {red }} \neq \rho_{\text {red }}^{2}\right)$ obtained by tracing out phases, or another subfluctuant variable. Indeed, a subfluctuant operator does not commute with the conjugate superfluctuant one, so that both operators cannot be simultaneously included in the complete set of observables characterizing a pure squeezed state.

In view of these preliminary remarks, we can say that the aim of this paper is twofold. First of all we want to compute the semiclassical Shannon-Wehrl entropy $[13,14]$ for a two-mode squeezed vacuum state, in order to show that also in that approach one recovers the result (2) for the large squeezing limit. This confirms that the entropy of a highly squeezed state is very robust to the particular scheme of coarse graining implemented (as discussed also in $[15,16]$ ).

In the second place we want to present a possible justification to the reduction of the density matrix in a superfluctuant basis [6,7], by interpreting such a reduction as the consequence of our lack of knowledge about the initial configuration which is the starting point of the squeezed evolution. To this purpose we shall represent the initial state, in general, as a statistical mixture of coherent states. This representation automatically defines a quantum von Neumann entropy associated to the initial mixture. This entropy however is constant, as the squeezed evolution is unitary, and then it does not contribute to the difference $\Delta S$ between final and initial entropy. However, if the statistical distribution satisfies (at least approximately) a principle of equipartition of the probabilities among the states of the mixture, then the off-diagonal terms of the density matrix turn out to be suppressed. An effective decoherence is thus produced, whose associated entropy in the superfluctuant basis is eventually amplified by the squeezed evolution.

The arguments presented in this paper are intended to apply to any process of pair production, described by a Bogoliubov transformation connecting the particle $\left\{b, b^{\dagger}\right\}$ and anti-particle $\left\{\tilde{b}, \tilde{b}^{\dagger}\right\}$ annihilation and creation $|i n\rangle$ operators to the $|o u t\rangle$ ones, $\left\{a, a^{\dagger}, \tilde{a}, \tilde{a}^{\dagger}\right\}$. Such a transformation reads, for each mode $k$,

$$
\begin{gathered}
a_{k}=c_{+}(k) b_{k}+c_{-}^{*}(k) \tilde{b}_{-k}^{\dagger} \\
\tilde{a}_{-k}^{\dagger}=c_{-}(k) b_{k}+c_{+}^{*}(k) \tilde{b}_{-k}^{\dagger}
\end{gathered}
$$


where the Bogoliubov coefficients $c_{ \pm}(k)$ satisfy $\left|c_{+}\right|^{2}-\left|c_{-}\right|^{2}=1$. By putting

$$
c_{+}(k)=\cosh r_{k}, \quad c_{-}^{*}(k)=e^{2 i \theta_{k}} \sinh r_{k}
$$

the transformation (3) can be rewritten as a unitary transformation,

$$
a_{k}=\Sigma_{k} b_{k} \Sigma_{k}^{\dagger}, \quad \tilde{a}_{-k}^{\dagger}=\Sigma_{k} \tilde{b}_{-k}^{\dagger} \Sigma_{k}^{\dagger}
$$

generated by the two-mode squeezing operator [8]

$$
\Sigma_{k}=\exp \left(z_{k}^{*} b_{k} \tilde{b}_{-k}-z_{k} b_{k}^{\dagger} \tilde{b}_{-k}^{\dagger}\right), \quad z_{k}=r_{k} e^{2 i \theta_{k}}
$$

Our discussion can thus be applied in general to any dynamic situation in which the initial vacuum state $|0\rangle$ evolves towards a final two-mode squeezed vacuum state, $|z\rangle=\Sigma_{k}|0\rangle$, characterized by a non-vanishing value of the squeezing parameter $r_{k}=\left|z_{k}\right|$ (related to the expectation value of the number of produced particles by $\left.\left\langle n_{k}\right\rangle=\left\langle z_{k}\left|b_{k}^{\dagger} b_{k}\right| z_{k}\right\rangle=\left\langle 0\left|a_{k}^{\dagger} a_{k}\right| 0\right\rangle=\left|c_{-}(k)\right|^{2}=\sinh ^{2} r_{k}\right)$.

It is important to note that in the context of such a dynamic evolution one can always define two operators $x$ and $\tilde{x}$, called superfluctuant, whose variances $(\Delta x)_{z}$, $(\Delta \tilde{x})_{z}$ are amplified with respect to their vacuum value $(\Delta x)_{0},(\Delta \tilde{x})_{0}$, namely $[8]$

$$
(\Delta x)_{0} \rightarrow(\Delta x)_{z}=(\Delta x)_{0} e^{r}, \quad(\Delta \tilde{x})_{0} \rightarrow(\Delta \tilde{x})_{z}=(\Delta \tilde{x})_{0} e^{r}
$$

where $(\Delta x)_{z}^{2}=\left\langle z\left|x^{2}\right| z\right\rangle-(\langle z|x| z\rangle)^{2}$ (and the same for $\tilde{x}$ ). The variance of the canonically conjugate operators $y, \tilde{y}$ is correspondingly squeezed, $(\Delta y)_{z}=$ $(\Delta y)_{0} e^{-r}=(\Delta \tilde{y})_{z}$. In terms of the superfluctuant variables the operators $b$ and $b^{\dagger}$ have the differential representation $[6,7,8]$

$$
\begin{aligned}
& b=\frac{i}{2} e^{i \theta}\left(x-i \tilde{x}+\partial_{x}-i \partial_{\tilde{x}}\right) \\
& \tilde{b}=\frac{i}{2} e^{i \theta}\left(x+i \tilde{x}+\partial_{x}+i \partial_{\tilde{x}}\right)
\end{aligned}
$$

(henceforth the mode index $k$ is to be understood, if not explicitly written, and any correlation among modes with different $|k|$ will be neglected, following the coarse graining approach of [2]). The normalized wave function $\psi_{z}$ for the twomode squeezed vacuum (such that $a \psi_{z}=0=\tilde{\alpha} \psi_{z}$ ) becomes, in the $(x, \tilde{x})$-space representation $[6,7]$,

$$
\psi_{z}(x, \tilde{x})=\langle x \tilde{x}|\Sigma| 0\rangle \equiv\langle x \tilde{x} \mid z\rangle=\left(\frac{\sigma}{\pi}\right)^{1 / 2} e^{-\frac{\sigma}{2}\left(x^{2}+\tilde{x}^{2}\right)},
$$




$$
\sigma=e^{-2 r}, \quad\langle z \mid z\rangle=1
$$

The squeezed evolution $|0\rangle \rightarrow|z\rangle=\Sigma|0\rangle$ may thus represented, in the superfluctuant basis, as a scaling transformation $x \rightarrow \sqrt{\sigma} x, \tilde{x} \rightarrow \sqrt{\sigma} \tilde{x}$, with real positive parameter $\sigma \leq 1$, related to the squeezing parameter $r$ according to eq.(9).

In order to compute the Shannon-Wehrl entropy for a squeezed state we recall that the classical entropy is defined, for any given phase-space distribution $f(q, p)$, as

$$
S_{c l}(f)=-\int d q d p f(q, p) \ln f(q, p)
$$

while the quantum (or von Neumann) entropy is defined in terms of the density operator $\rho$ as

$$
S_{q}(\rho)=-\operatorname{Tr} \rho \ln \rho
$$

For a pure state, in particular, $\rho^{2}=\rho$ and $S_{q}=0$. Suppose now that we want to compute, for a given quantum state represented by $\rho$, its entropy in the "semiclassical" limit. It is well known that a possible procedure to obtain the semiclassical limit of a quantum observable is to compute the expectation value of that observable in the basis of the coherent states $|\alpha\rangle$. According to Wehrl [14], a natural semiclassical phase-space distribution associated to $\rho$ is thus given by

$$
f(\alpha)=\frac{1}{\pi}\langle\alpha|\rho| \alpha\rangle
$$

(the so-called Glauber distribution function [17]), which leads to define the semiclassical (Wehrl) entropy $S_{W}$ as

$$
S_{W}=S_{c l}(f(\alpha))
$$

For a two-mode squeezed state $|z\rangle$ (see $[18,19]$ for the one-mode case) we have, in particular,

$$
f(\alpha, \tilde{\alpha})=\frac{1}{\pi^{2}}\langle\alpha \tilde{\alpha} \mid z\rangle\langle z \mid \alpha \tilde{\alpha}\rangle
$$

where the coherent states $|\alpha \tilde{\alpha}\rangle$ satisfy

$$
b|\alpha \tilde{\alpha}\rangle=\alpha|\alpha \tilde{\alpha}\rangle, \quad \tilde{b}|\alpha \tilde{\alpha}\rangle=\tilde{\alpha}|\alpha \tilde{\alpha}\rangle
$$

(the operators $b, \tilde{b}$ are defined in eq.(8); their corresponding eigenvalues are complex numbers, $\left.\alpha=\alpha_{1}+i \alpha_{2}, \tilde{\alpha}=\tilde{\alpha}_{1}+i \tilde{\alpha}_{2}\right)$. By expanding in the superfluctuant basis $|x \tilde{x}\rangle$ one easily obtains

$$
\langle x \tilde{x} \mid \alpha \tilde{\alpha}\rangle=\frac{1}{\sqrt{\pi}} \exp \left[-\frac{1}{2}(\Re k)^{2}-\frac{1}{2}(\Re \tilde{k})^{2}+k x+\tilde{k} \tilde{x}-\frac{1}{2}\left(x^{2}+\tilde{x}^{2}\right)\right],
$$




$$
\langle\alpha \tilde{\alpha} \mid \alpha \tilde{\alpha}\rangle=1
$$

where $\Re z$ denotes the real part of $z$, and

$$
k=-i e^{-i \theta}(\alpha+\tilde{\alpha}), \quad \tilde{k}=-e^{-i \theta}(\tilde{\alpha}-\alpha)
$$

Moreover

$$
\begin{aligned}
& f(\alpha, \tilde{\alpha})=\frac{1}{\pi^{2}} \int d x d \tilde{x} d x^{\prime} d \tilde{x}^{\prime} \psi_{z}(x \tilde{x}) \psi_{z}^{*}\left(x^{\prime} \tilde{x}^{\prime}\right)\langle\alpha \tilde{\alpha} \mid x \tilde{x}\rangle\left\langle x^{\prime} \tilde{x}^{\prime} \mid \alpha \tilde{\alpha}\right\rangle= \\
& =\frac{1}{\pi^{2} \cosh ^{2} r} \exp \left[-\tanh r\left(\alpha \tilde{\alpha} e^{-2 i \theta}+\alpha^{*} \tilde{\alpha}^{*} e^{2 i \theta}\right)-|\alpha|^{2}-|\tilde{\alpha}|^{2}\right]
\end{aligned}
$$

With this Glauber distribution, the semiclassical Wehrl entropy (13) for the twomode squeezed vacuum is then

$$
S_{W}=-\int d^{2} \alpha d^{2} \tilde{\alpha} f(\alpha, \tilde{\alpha}) \ln f(\alpha, \tilde{\alpha})=2+2 \ln \pi+2 \ln \cosh r
$$

The same result holds for a squeezed-coherent state $\left|z \alpha_{0} \tilde{\alpha}_{0}\right\rangle=\Sigma\left|\alpha_{0} \tilde{\alpha}_{0}\right\rangle$, obtained by applying a squeezing transformation to the "displaced" vacuum $\left|\alpha_{0} \tilde{\alpha}_{0}\right\rangle=$ $D\left(\alpha_{0}\right) D\left(\tilde{\alpha}_{0}\right)|0\rangle$, where $D(\alpha)=\exp \left(\alpha b^{\dagger}-\alpha^{*} b\right)$ is the Glauber displacement operator.

It is important to note that for $r>>1$ this semiclassical entropy reproduces the large squeezing behavior of eq.(2), previously obtained [1,3-7,12] with different approaches to the entropy of a particle production process. We also note that the entropy (19) is exactly the sum of two "marginal" entropies,

$$
S_{W}=S_{\alpha_{1} \tilde{\alpha}_{1}}^{W}+S_{\alpha_{2} \tilde{\alpha}_{2}}^{W}
$$

obtained by decomposing the probability distribution $f(\alpha, \tilde{\alpha})$ in the complex $\alpha, \tilde{\alpha}$ planes, and by tracing out one of the two cartesian variables,

$$
\begin{gathered}
S_{\alpha_{1} \tilde{\alpha}_{1}}^{W}=-\int d \alpha_{1} d \tilde{\alpha}_{1} f\left(\alpha_{1}, \tilde{\alpha}_{1}\right) \ln f\left(\alpha_{1}, \tilde{\alpha}_{1}\right), \quad f\left(\alpha_{1}, \tilde{\alpha}_{1}\right)=\int d \alpha_{2} d \tilde{\alpha}_{2} f(\alpha, \tilde{\alpha}) \\
S_{\alpha_{2} \tilde{\alpha}_{2}}^{W}=-\int d \alpha_{2} d \tilde{\alpha}_{2} f\left(\alpha_{2}, \tilde{\alpha}_{2}\right) \ln f\left(\alpha_{2}, \tilde{\alpha}_{2}\right), \quad f\left(\alpha_{2}, \tilde{\alpha}_{2}\right)=\int d \alpha_{1} d \tilde{\alpha}_{1} f(\alpha, \tilde{\alpha})
\end{gathered}
$$

This is a non-trivial result, as it implies that the information-theoretical Araki-Lieb inequality [20], $S \leq S_{\alpha_{1} \tilde{\alpha}_{1}}+S_{\alpha_{2} \tilde{\alpha}_{2}}$, is maximized both by the two-mode squeezed vacuum and by a squeezed-coherent state, in agreement with the properties of one-mode squeezed states [18]. 
According to eq.(19), the semiclassical entropy associated to a pure squeezed state $|z\rangle$ is thus non-vanishing, in spite of the fact that $\rho=|z\rangle\langle z|=\rho^{2}$ and $S_{q}(\rho)=$ 0 . This occurs because an effective reduction of the quantum density matrix is implicit in the semiclassical limit, as even a pure state is seen, classically, as a disordered one. However, the coarse graining associated to the semiclassical limit cannot give us explicit information about the physical origin of the decoherence mechanism, which can only be described in the context of some dynamic model for the quantum to classical transition (see also [21]). For a squeezed state such a model must account, in particular, for the fact that some degree of freedom of the system is made dynamically less relevant than others for what concerns macroscopic observations.

Let us consider now the possibility of defining also a von Neumann entropy for the squeezing process, in terms of a properly reduced density operator. In the approach of Refs. [5-7] the reduction was performed by neglecting the off-diagonal elements of the density matrix in a superfluctuant representation. The use of such a representation is justified because the squeezed density operator can only be reconstructed, observationally, by measuring superfluctuant variables and their momenta. But is the reduction a formally justified procedure?

The answer is certainly not for the pure squeezed vacuum state (9), whose density matrix in the superfluctuant representation

$$
\begin{gathered}
\rho_{z}\left(x, x^{\prime}, \tilde{x}, \tilde{x}^{\prime}\right)=\langle x \tilde{x} \mid z\rangle\left\langle z \mid x^{\prime} \tilde{x}^{\prime}\right\rangle=\psi_{z}(x, \tilde{x}) \psi_{z}^{*}\left(x^{\prime}, \tilde{x}^{\prime}\right)= \\
=\frac{\sigma}{\pi} \exp \left[-\frac{\sigma}{2}\left(x^{2}+x^{\prime 2}+\tilde{x}^{2}+\tilde{x}^{\prime 2}\right)\right]
\end{gathered}
$$

is perfectly symmetric in the $\left(x, x^{\prime}\right)$ and $\left(\tilde{x}, \tilde{x}^{\prime}\right)$ planes. The same answer holds for a pure squeezed number and squeezed-coherent state. On the contrary, the reduction would be naturally justified if the off-diagonal matrix elements would be suppressed, $\left|\rho_{z}(x, x)\right|>>\left|\rho_{z}\left(x, x^{\prime} \neq x\right)\right|$ (and the same for $\tilde{x}$ ), for any given value of the squeezing parameter. It is thus interesting to note that such requirement can be satisfied when the initial state is not pure, but is represented by a mixture, whose statistical weights tend to be equally distributed among all the states of the mixture.

Suppose to start, in fact, from an initial configuration more general than the vacuum, represented by an ensemble of states which, like the vacuum, minimize however the quantum fluctuations $\Delta x, \Delta \tilde{x}$ ( and their conjugate $\Delta y, \Delta \tilde{y}$ ). This corresponds to an initial density operator which can be expressed in terms of the 
coherent states (15), (16) as

$$
\rho_{i}=\int d^{2} \alpha d^{2} \tilde{\alpha} P(\alpha, \tilde{\alpha})|\alpha \tilde{\alpha}\rangle\langle\alpha \tilde{\alpha}|
$$

where the statistical weights $P \geq 0$ satisfy the normalization condition

$$
\int d^{2} \alpha d^{2} \tilde{\alpha} P(\alpha, \tilde{\alpha})=1
$$

The squeezed evolution leads then to a final density operator, $\rho_{f}=\Sigma \rho_{i} \Sigma^{\dagger}$, which in the superfluctuant basis is explicitly represented by

$$
\rho_{f}\left(x, x^{\prime}, \tilde{x}, \tilde{x}^{\prime}\right)=\int d^{2} \alpha d^{2} \tilde{\alpha} P(\alpha, \tilde{\alpha}) \psi_{z \alpha \tilde{\alpha}}(x, \tilde{x}) \psi_{z \alpha \tilde{\alpha}}^{*}\left(x^{\prime}, \tilde{x}^{\prime}\right)
$$

Here $\psi_{z \alpha \tilde{\alpha}}(x, \tilde{x})$ is the squeezed-coherent wave function, representing in the $(x, \tilde{x})$ space the eigenfunctions of $\{a, \tilde{a}\}$ with eigenvalues $\{\alpha, \tilde{\alpha}\}$, namely

$$
\begin{gathered}
\psi_{z \alpha \tilde{\alpha}}(x, \tilde{x})=\langle x \tilde{x} \mid z \alpha \tilde{\alpha}\rangle=\langle x \tilde{x}|\Sigma| \alpha \tilde{\alpha}\rangle= \\
=\sqrt{\frac{\sigma}{\pi}} \exp \left[-\frac{1}{2}(\Re k)^{2}-\frac{1}{2}(\Re \tilde{k})^{2}+\sqrt{\sigma}(k \sigma+\tilde{k} \tilde{x})-\frac{\sigma}{2}\left(x^{2}+\tilde{x}^{2}\right)\right]
\end{gathered}
$$

$\left(k, \tilde{k}\right.$ are defined in eq.(17)). Note that, for $\rho_{i} \neq \rho_{i}^{2}$, there is a non-vanishing quantum entropy, $S_{q}=-\int d^{2} \alpha d^{2} \tilde{\alpha} P(\alpha, \tilde{\alpha}) \ln P(\alpha \tilde{\alpha})$, naturally associated to the initial mixture. Such an entropy, however, is constant throughout the whole process of squeezing $\rho_{i} \rightarrow \rho_{f}$ (which describes a unitary evolution), so that it cannot contribute to the overall entropy difference $\Delta S=S_{f}=S_{i}$.

The choice of the vacuum as initial condition corresponds to the assumption $P(\alpha, \tilde{\alpha})=\delta^{2}(\alpha) \delta^{2}(\tilde{\alpha})$ in eq.(25). We shall consider here an initial distribution which, instead of being infinitely peaked upon the vacuum, tends to be spread rather uniformly over a wide range of states, so as to reflect our lack of knowledge about the initial conditions. We shall assume, however, that such a distribution is always centered around the vacuum, and we shall conveniently translate the $(x, \tilde{x})$ frame in such a way that $\langle z \alpha \tilde{\alpha}|x| z \alpha \tilde{\alpha}\rangle=0=\langle z \alpha \tilde{\alpha}|\tilde{x}| z \alpha \tilde{\alpha}\rangle$ (which implies $\Re k=$ $0=\Re \tilde{k}$, see eq.(26)). The simplest example satisfying the above requirements is a normalized square-like distribution, in which the probability of a state $|\alpha \tilde{\alpha}\rangle$ is constant and equal to $1 / L$ for $|\alpha|,|\tilde{\alpha}|<L / 2$, and vanishing for $|\alpha|,|\tilde{\alpha}|>L / 2$, namely

$$
\int d^{2} \alpha d^{2} \tilde{\alpha} P(\alpha, \tilde{\alpha})=d \alpha_{1} d \alpha_{2} d \tilde{\alpha}_{1} d \tilde{\alpha}_{2} \delta\left(\alpha_{1}-\tilde{\alpha}_{1}\right) \delta\left(\alpha_{2}+\tilde{\alpha}_{2}\right) \times
$$




$$
\times \frac{1}{L^{2}}\left[\theta\left(\alpha_{1}+L / 2\right)-\theta\left(\alpha_{1}-L / 2\right)\right]\left[\theta\left(\alpha_{2}+L / 2\right)-\theta\left(\alpha_{2}-L / 2\right)\right]
$$

Here $\theta$ is the Heaviside step function, and the two delta distributions have been inserted to implement the conditions $\langle x\rangle=0=\langle\tilde{x}\rangle$; we have also rescaled the variables $\alpha, \tilde{\alpha}$ so as to absorb the overall phase $e^{-i \theta}$ which does not contribute to the integration over the whole complex plane.

With this probability distribution the squeezed matrix (25) becomes

$$
\rho_{f}\left(x, x^{\prime}, \tilde{x}, \tilde{x}^{\prime}\right)=\frac{\sigma}{\pi} \frac{\sin \left[L \sqrt{\sigma}\left(x^{\prime}-x\right)\right]}{L \sqrt{\sigma}\left(x^{\prime}-x\right)} \frac{\sin \left[L \sqrt{\sigma}\left(\tilde{x}-\tilde{x}^{\prime}\right)\right]}{L \sqrt{\sigma}\left(\tilde{x}-\tilde{x}^{\prime}\right)} e^{-\frac{\sigma}{2}\left(x^{2}+x^{\prime 2}+\tilde{x}^{2}+\tilde{x}^{\prime 2}\right)}
$$

and it is immediately evident that, for large enough $L$, the off-diagonal terms $x \neq x^{\prime}, \tilde{x} \neq \tilde{x}^{\prime}$ are suppressed with respect to the diagonal ones. Such a suppression grows with $L$, namely with the number of states which are considered equiprobable in the initial distribution, i.e. with the growth of our "ignorance" about the initial configuration. For large $L$ we may thus approximate the squeezed matrix with the diagonal elements only, and in particular we can formally define in this basis a (normalized) reduced density matrix, in the limit $L \rightarrow \infty$ (at finite $\sigma$ ), as

$$
\begin{gathered}
\rho_{\text {red }}\left(x, x^{\prime}, \tilde{x}, \tilde{x}^{\prime}\right)=\lim _{L \rightarrow \infty}\left\{L^{2} \sigma \rho_{f}\left(x, x^{\prime}, \tilde{x}, \tilde{x}^{\prime}\right)\right\}= \\
=\frac{\sigma}{\pi} e^{-\sigma\left(x^{2}++\tilde{x}^{2}\right)} \delta\left(x-x^{\prime}\right) \delta\left(\tilde{x}-\tilde{x}^{\prime}\right)
\end{gathered}
$$

This is exactly the representation in the $(x, \tilde{x})$ basis of the reduced operator

$$
\rho_{\text {red }}=\int d x d \tilde{x}\left|\psi_{z}(x, \tilde{x})\right|^{2}|x \tilde{x}\rangle\langle x \tilde{x}|
$$

previously used in Refs.[6,7].

Note that $\operatorname{Tr} \rho_{\text {red }}=1$, but $\rho_{\text {red }}^{2} \neq \rho_{\text {red }}$. This reduction leads then to a quantum entropy $S_{q}\left(\rho_{\text {red }}\right)$ which, unlike the entropy of the mixture $S_{q}\left(\rho_{f}\right)$, depends on $\sigma$, and in particular grows with the degree of squeezing. By taking the difference between final and initial entropy we find indeed the result of eq.(2),

$$
\Delta S=\left(\operatorname{Tr} \rho_{\text {red }} \ln \rho_{\text {red }}\right)_{\sigma=1}-\left(\operatorname{Tr} \rho_{\text {red }} \ln \rho_{r e d}\right)_{\sigma}=2 r
$$

The accuracy of this estimate in our context becomes larger and larger as the initial probability distribution becomes flatter, so as to share probabilities among a larger number of states. It is also important to note that if, for a given $L$, the reduced matrix represent a good approximation to the exact matrix (28) at large 
squeezing $(\sigma>>1)$, then the approximation is even better as we go back in time towards the initial configuration, where $\sigma=1$.

We may thus conclude that the effective reduction of the squeezed density operator, introduced "ad hoc" in previous papers [6,7], can be formally justified through a limiting procedure based on an appropriate mixture of coherent states, assumed to represent the initial configuration. The corresponding entropy growth may thus physically interpreted as an amplification, due to the squeezed evolution, of our ignorance about the initial conditions, in a context in which we have no motivation to single out some preferred initial state, and we cannot reconstruct the initial state through final measurements of superfluctuant variables only. Such an interpretation seems to be particularly appropriate in the context of cosmological perturbation theory, where the amplification of the background fluctuations is computed starting from an initial state which is left unspecified except for the requirement of minimizing the quantum fluctuations, and which can thus be properly represented as a general mixture of coherent states.

\section{Acknowledgments}

One of us (M. Gasperini) wishes to thank D. Boyanovsky for rising stimulating questions that motivated in part the work reported here. We are also grateful to G. Veneziano for many helpful discussions.

\section{References}

1. B. L. Hu and D. Pavon, Phys. Lett. B 180, 329 (1986)

2. B. L. Hu and H. E. Kandrup, Phys. Rev. D 35, 1776 (1987);

H. E. Kandrup, Phys. Rev. D 37, 3505 (1988);

for a recent review on cosmological particle production see also B. L. Hu, G. Kang and A. Matacz, Int. J. Mod. Phys. A 9, 991 (1994)

3. R. Brandenberger, V. Mukhanov and T. Prokopec, Phys. Rev. Lett. 69, 3606 (1992)

4. R. Brandenberger, V. Mukhanov and T. Prokopec, Phys. Rev. D 48, 2443 (1993) 
5. T. Prokopec, Class. Quantum Grav. 10, 2295 (1993)

6. M. Gasperini and M. Giovannini, Phys. Lett. B 301, 334 (1993)

7. M. Gasperini and M. Giovannini, Class. Quantum Grav. 10, L133 (1993)

8. B. L. Shumaker, Phys. Rep. 135, 317 (1986);

J. Grochmalicki and M. Lewenstein, Phys. Rep. 208, 189 (1991)

9. B. L. Hu, Phys. Rev. D 9, 3263 (1974)

10. L. P. Grishchuk and Y. V. Sidorov, Phys. Rev. D 42, 3413 (1990);

L. P. Grishchuk and L. Solokhin, Phys. Rev. D 43, 2566 (1991)

11. L. P. Grishchuk, Zh. Eksp. Teor. Fiz. 67, 825 (1975)

12. A. Matacz, Phys. Rev. D 49, 788 (1994)

13. C. E. Shannon and W. Weaver, "The mathematical theory of communication" (Univ. of Illinois Press, Urbana, IL, 1963)

14. A. Wehrl, Rev. Mod. Phys. 50, 221 (1978)

15. E. Keski-Vakkuri, Phys. Rev. D49, 2122, (1994)

16. M. Kruczenski, L. E. Oxman and M. Zaldarriaga, "Large squeezing behavior of cosmological entropy generation", Preprint gr-qc/9403024

17. R. London and P. L. Knight, J. Mod. Opt. 34, 709 (1987)

18. C. Keitel and K. Wodkiewicz, Phys. Lett. A 167, 151 (1992)

19. H. Rosu and M. Reyes, "Shannon-Wehrl entropy for cosmological and blackhole squeezing", Preprint gr-qc/9406001

20. H. Araki and E. Lieb, Commun. Math. Phys. 18, 160 (1970)

21. W. H. Zurek, S. Habib and J. P. Paz, Phys. Rev. Lett. 70, 1187 (1993) 